\title{
Comparison of the efficacy of PSI, CURB-65, CALL and BCRSS in predicting prognosis and mortality in COVID-19 patients
}

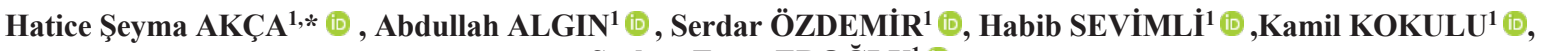 \\ Serkan Emre EROĞLU ${ }^{1}$ (i)
}

${ }^{1}$ Department of Emergency Medicine, University of Health Sciences, Ümraniye Training and Research Hospital, Istanbul, Turkey

\begin{abstract}
\begin{tabular}{ccccc}
\hline Received: 16.02 .2021 & $\bullet$ & Accepted/Published Online: 27.02 .2021 & $\bullet$ & Final Version: 30.08 .2021 \\
\hline
\end{tabular}
\section{Abstract}

This study aimed to determine whether the PSI, CURB-65, CALL and BCRSS had any superiority over each other as a prognostic determinant in patients with COVID-19. This prospective cohort study included patients over 18 years of age that presented to the emergency department between May 12 and August 12, 2020 and had a positive COVID-19 polymerase chain reaction (PCR) test. The PSI, CURB-65, CALL and BCRS scores were calculated. SPSS version 22 was used for all statistical analyses. A total of 213 patients with a positive COVID-19 PCR result were included in the study. The total 30-day mortality rate was determined as $14.08 \%$. PSI, CURB-65, CALL and BCRSS had a statistically significant relationship with mortality $(\mathrm{p}<0.001)$. The best parameter in predicting mortality was determined as PSI (area under the curve: 0.900 ; 95\% CI: 0.972-0.828). A positive correlation was found between each scoring system, both with the length of hospital stay (PSI, CURB-65, CALL and BCRSS: $\mathrm{r}=0.696, \mathrm{p}=0 ; \mathrm{r}=0.621, \mathrm{p}=0 ; \mathrm{r}=0.75, \mathrm{p}=0$; and $\mathrm{r}=0.666, \mathrm{p}=0$, respectively). Scoring systems, which include comorbidity, vital signs as well as laboratory, imaging findings, will be more effective than other scoring systems in determining the mortality in patients with covid-19.
\end{abstract}

Keywords: BCRSS, CALL, COVID-19, CURB-65, PSI

\section{Introduction}

A previously unidentified viral pneumonia was detected in Wuhan in December 2019 and named SARS-CoV-2 or COVID-19. It rapidly spread to other countries within a few months and was soon declared a pandemic. In Turkey, the first cases were seen in the March 11, 2020, and the number of cases reached a peak level toward the end of April and started to decrease in late May. An increasing trend started to be seen again as of 15 June, and the increase of 1,000-1,500 cases per day continued until August 30. A daily increase of 100-500 cases was observed from August 30 to December 10. The number of cases has been showing a decreasing trend since December 10 (1). The Pneumonia Severity Index (PSI), CURB-65 (cased on the presence or absence of the following criteria: new confusion, urea $>7 \mathrm{mmol} / \mathrm{l}$, respiratory rate $>$ $30 / \mathrm{min}$, systolic blood pressure $<90 \mathrm{~mm} \mathrm{Hg}$ or diastolic blood pressure $\leq 60 \mathrm{~mm} \mathrm{Hg}$, and age $\geq 65$ years) and CALL (comorbidity, age (years), lymphocyte, lactate dehydrogenase) scoring systems as predictors of prognosis and mortality in patients with pneumonia (2-4). It remains unclear whether these scoring systems can also be determinant in the prognosis and mortality of patients with COVID-19. The Brescia-COVID Respiratory Severity Scale/Algorithm (BCRSS) has been previously used in
COVID-19 cases but there are only a limited number of studies evaluating its efficacy (5).

Like the situation in other parts of the world, after the COVID-19 pandemic reached Turkey, we started to apply current algorithms based on frequently encountered symptoms and clinical findings to predict the prognosis of these patients. However, patients' outcomes sometimes differ from what is expected. Therefore, we planned to conduct a comprehensive study on whether pneumonia scoring systems or different severity indicators were superior to each other in predicting prognosis in patients with COVID-19 and share the results with the literature. This study aimed to show whether PSI, CURB-65, CALL and BCRSS had any superiority over each other as prognosis determinants in COVID-19 cases.

\section{Materials and methods}

\subsection{Study design}

This prospective cohort study was conducted in the emergency department of Ümraniye Training and Research Hospital, which includes a COVID outpatient clinic. This emergency department is a well-equipped clinic serving an average of 500,000 patients every year with green, yellow and red zones and a resuscitation unit. 


\subsection{Patient population}

The study included patients over 18 years of age, who presented to the emergency department with different symptoms between May 12 and August 12, 2020 and had a positive COVID-19 polymerase chain reaction (PCR) test. Patients without COVID-19 PCR results and those concurrently diagnosed with other diseases in addition to COVID-19 were not included in the study.

\subsection{Data collection}

The presenting symptoms, history, vital signs, examination findings, and laboratory test results of the patients were recorded. The patients with and without computed tomography findings were grouped. At the time of presented, the patients were categorized according to the algorithm of the World Health Organization and the Turkish Ministry of Health $(1,6)$. Contact history was questioned. According to the outcome, the patients were divided into three groups as those that were discharged, those that were hospitalized, and those that were admitted to the intensive care unit (ICU). The length of hospital stay, and 30-day mortality were recorded. According to the clinical findings, the PSI, CURB-65, CALL and BCRSS values were obtained and compared in terms of their efficacy in predicting prognosis and mortality. The primary outcome of this study was the comparison of the efficacy of the PSI, CURB-65, CALL and BCRSS in predicting mortality in patients with COVID-19, and the secondary outcome was to determine the superiority of these scoring systems over each in terms of the length of hospital stay.

\subsection{Statistical analyses}

Statistical analysis was performed using SPSS version 22.0. The conformance of variables to normal distribution was examined by visual (histogram and probability graphs) and analytical methods (Kolmogorov-Smirnov). The chi-square test was conducted to evaluate the relationship between categorical data. The Mann-Whiney $U$ test was used to compare non-parametric numerical data between two groups. If there were more than two groups, the Kruskal-Wallis test was used to compare non-parametric numerical data. We also formed a characteristic curve (ROC) for 30-day mortality and obtained the area under the curve (AUC) for individual variables. Spearman's correlation analysis was undertaken to determine the relationship between the PSI, CURB-65, CALL and BCRSS systems and length of hospital stay. While investigating the associations between non-normally distributed and/or ordinal variables, the correlation coefficients and their significance were calculated using the Spearman test. A $p$ value of $<0.05$ was accepted as statistically significant.

\subsection{Ethics}

For the study, ethical approval was obtained from the local clinical research ethics committee of our hospital (date: April 28, 2020, number: B.10.1.TKH.4.34.H.GP.0.01/123). Patients with a sufficient level of consciousness and the relatives of those who did not have sufficient consciousness were invited to participate in the study. An informed consent form was signed by the patients or their relatives who accepted to participate in the study.

\section{Results}

The study included a total of 213 patients $(53.1 \%$ male) with a positive COVID-19 PCR result. The mean age mean was $46.54 \pm 19.52$ years. It was observed that increasing age increased 30-day mortality $(\mathrm{p}<0.001)$. Total 30 -day mortality rate was determined as $14.08 \%$. Of the patients that died, $56.7 \%$ were male. Within the first 24 hours, $29.01 \%$ of the patients were admitted to inpatient services and $15.02 \%$ to ICU. On first physical examination, a statistically significant relationship was found between the respiratory rate per minute and oxygen saturation at the time of the first measurement and mortality $(\mathrm{p}<0.001)$. A high respiratory rate and a low saturation value increased mortality. When comorbidities were examined, the mortality rate was higher among the patients with hypertension, congestive heart failure (CHF) $(\mathrm{p}<0.001)$, chronic obstructive pulmonary disease (COPD) $(\mathrm{p}=0.001)$, chronic kidney disease (CKD) and coronary artery disease $(\mathrm{CAD})(\mathrm{p}<0.001)$. In addition, the presence of confusion, cough, and weakness $(p<0.001)$, shortness of breath $(p=0.008)$, myalgia $(p=0.024)$ and sore throat $(p<0.005)$ increased mortality. Mortality was also higher in the patients with a history of contact $(p<0.001)$. It was observed that prolonged hospital stay was associated with increased mortality $(p<0.001)$. Increased CURB-65 and PSI values indicated higher mortality $(\mathrm{p}<0.001)$ (Table.1). The relationships between demographic data, symptoms, physical examination findings and mortality are shown in Table 1.

We divided the BCRSS and CALL scores into classes using the special grouping method for these systems and determined that as the BCRSS level or CALL class increased, there was an increase in mortality at a statistically significant level $(\mathrm{p}<0.001)$ (Table 2).

The Spearman correlation test was performed between PSI, CURB-65, CALL and BCRSS and age, saturation value, and length of hospital stay. Saturation was negatively correlated with CURB-65, CALL, and BCRSS. The scores increased as the saturation value decreased, and age increased. Other data were positively correlated with each other. Each scoring system was correlated with each other, as well as with the length of hospital stay and age (correlation between the length of hospital stay and PSI, CURB-65, CALL and BCRSS: $\mathrm{r}=0.696, \mathrm{p}=0 ; \mathrm{r}=0.621, \mathrm{p}=0 ; \mathrm{r}=0.75, \mathrm{p}=0$; and $\mathrm{r}=0.666, \mathrm{p}=0$, respectively) (Table 3 ). We conducted the receiver operating characteristic (ROC) curve analysis of the relationship between the four scoring systems with mortality within 30 days of presentation to the emergency to calculate the cut-off values of these scoring systems in predicting mortality. 
Table 1. Relationship of demographic characteristics, symptoms, physical examination findings and scoring systems with mortality

\begin{tabular}{|c|c|c|c|c|}
\hline & Total & Survivor & Non-survivor & $\mathrm{P}$ value \\
\hline Age $($ mean \pm SD) & $46.54 \pm 19.52(18-95)$ & $42.55 \pm 16.87(18-95)$ & $70.87 \pm 16.95(22-92)$ & $<0.001$ \\
\hline Gender (n, \%) & & & & 0.669 \\
\hline Female & $100(46.9 \%)$ & $87(47.5 \%)$ & $13(43.3 \%)$ & \\
\hline Male & $113(53.1 \%)$ & $96(52.5 \%)$ & $17(56.7 \%)$ & \\
\hline Systolic TA (mean \pm SD) & $121.53 \pm 14.61(62-188)$ & $121.44 \pm 10.8(90-164)$ & $122.10 \pm 28.75(62-188)$ & 0.888 \\
\hline Diastolic TA (mean \pm SD) & $73.71 \pm 10.08(34-118)$ & $74.32 \pm 8.79(45-118)$ & $70.03 \pm 15.56(34-102)$ & 0.056 \\
\hline Respiratory rate (mean \pm SD & $19.33 \pm 5.35(12-40)$ & $18.24 \pm 3.78(12-40)$ & $25.97 \pm 8.18(15-40)$ & $<0.001$ \\
\hline Fever $($ mean $\pm \mathrm{SD})$ & $36.5 \pm 0.37(36-39)$ & $36.5 \pm 0.38(36-39)$ & $36.5 \pm 0.29(36-38)$ & 0.82 \\
\hline Saturation (mean \pm SD & $95.23 \pm 6.97(50-100)$ & $96.86 \pm 3.65(71-100)$ & $85.30 \pm 12.37(50-99)$ & $<0.001$ \\
\hline Length of hospitalstay (days) & $3.98 \pm 6.8(0-35)$ & $2.98 \pm 5.49(0-33)$ & $10.07 \pm 10.24(0-35)$ & $<0.001$ \\
\hline \multicolumn{5}{|l|}{ Comorbidities (n, \%) } \\
\hline Hypertension & $49(23 \%)$ & $30(16.4 \%)$ & $19(63.3 \%)$ & $<0.001$ \\
\hline Diabetesmellitus & $32(15 \%)$ & $25(13.7 \%)$ & $7(23.3 \%)$ & 0.169 \\
\hline COPD & $7(3.3 \%)$ & $3(1.6 \%)$ & $4(13.3 \%)$ & 0.001 \\
\hline $\mathrm{CCF}$ & $9(4.2 \%)$ & $3(1.6 \%)$ & $6(20 \%)$ & $<0.001$ \\
\hline CKD & $9(4.2 \%)$ & $5(2.7 \%)$ & $4(13.3 \%)$ & 0.029 \\
\hline CAD & $11(5.2 \%)$ & $6(3.3 \%)$ & $5(16.7 \%)$ & 0.009 \\
\hline Contacthistory $(\mathrm{n}, \%)$ & $115(54 \%)$ & $108(59 \%)$ & $7(23.3 \%)$ & $<0.001$ \\
\hline \multicolumn{5}{|l|}{ Symptoms $(\mathrm{n}, \%)$} \\
\hline Fever & $69(32.4 \%)$ & $63(34.4 \%)$ & $6(20 \%)$ & 0.118 \\
\hline Confusion & $21(9.9 \%)$ & $7(3.8 \%)$ & $14(46.7 \%)$ & $<0.001$ \\
\hline Shortness of breath & $69(32.4 \%)$ & $53(29 \%)$ & $16(53.3 \%)$ & 0.008 \\
\hline Cough & $114(53.5 \%)$ & $107(58.5 \%)$ & $7(23.3 \%)$ & $<0.001$ \\
\hline Myalgia & $66(31 \%)$ & $62(33.9 \%)$ & $4(13.3 \%)$ & 0.024 \\
\hline Sorethroat & $27(12.7 \%)$ & $27(14.8 \%)$ & 0 & 0.024 \\
\hline Inabilitytotaste & $13(6.1 \%)$ & $12(6.6 \%)$ & $1(3.3 \%)$ & 0.494 \\
\hline Inabilitytosmell & $11(5.2 \%)$ & $10(5.5 \%)$ & $1(3.3 \%)$ & 0.625 \\
\hline Weakness & $75(35.2 \%)$ & $51(27.9 \%)$ & $24(80 \%)$ & $<0.001$ \\
\hline SBP<90 mmhg (n\%) & $4(1.9 \%)$ & 0 & $4(13.3 \%)$ & $<0.001$ \\
\hline $\mathrm{DBP}<60 \mathrm{mmhg}(\mathrm{n} \%)$ & $12(5.6 \%)$ & $8(4.4 \%)$ & $4(13.3)$ & 0.122 \\
\hline Respiratory rate $>30 / \min (\mathrm{n} \%)$ & $10(4.7 \%)$ & $3(1.6 \%)$ & $7(23.3)$ & $<0.001$ \\
\hline CT finding ( $\mathrm{n} \%)$ & $116(54.5 \%)$ & $90(49.2 \%)$ & $26(86.7 \%)$ & $<0.001$ \\
\hline CURB-65 (mean \pm SD & $1 \pm 1.07(0-5)$ & $0.74 \pm 0.75(0-3)$ & $2.6 \pm 1.35(0-5)$ & $<0.001$ \\
\hline BCRSS (mean \pm SD & $0.49 \pm 1.03(0-4)$ & $0.23 \pm 0.65(0-4)$ & $2.07 \pm 1.48(0-4)$ & \\
\hline CALL $($ mean \pm SD & $5.97 \pm 2.72(0-13)$ & $5.36 \pm 2.14(0-12)$ & $9.7 \pm 2.91(4-13)$ & \\
\hline PSI (mean \pm SD & $58.05 \pm 45.93(8-253)$ & $45.67 \pm 28.93(8-147)$ & $133.57 \pm 57.46(22-253)$ & $<0.001$ \\
\hline
\end{tabular}

COPD: chronic obstructive pulmonary disease, CCF: congestive cardiac failure, CKD: chronic kidney disease, CAD:coronary artery disease,

SBP: systolic blood pressure, DBP: diastolic blood pressure

Table 4 presents the cut-off, sensitivity, specificity, 95\% confidence interval and area under the curve (AUC) values. The cut-off values of each scoring system were statistically significant in predicting mortality, and the AUC values were at a good level. The best parameter in predicting mortality

Table 2. Relationship of BCRSS and CALL with mortality was determined to be PSI with an AUC value of 0.900 (95\% CI: 0.972-0.828). The length of hospital stay was a relatively poor determinant of mortality, with an AUC value of 0.790 (95\% CI: 0.874-0.706). The remaining parameters had similar efficacy in predicting mortality (Table 4) (Fig.1).

\begin{tabular}{|l|l|l|l|}
\hline & Total & Survivor & Non-survivor \\
\hline BCRSS & & & \\
\hline Level 0 & $161(75.6 \%)$ & $155(84.7 \%)$ & $6(20 \%)$ \\
\hline Level 1 & $24(11.3 \%)$ & $18(9.8 \%)$ & $6(20.0 \%)$ \\
\hline Level 2 & $12(5.6 \%)$ & $7(3.8 \%)$ & $5(16.7 \%)$ \\
\hline Level 3 & $7(3.3 \%)$ & $1(0.5 \%)$ & $6(20.0 \%)$ \\
\hline Cevel 4 & $9(4.2 \%)$ & $2(1.1 \%)$ & $7(23.3 \%)$ \\
\hline ClasL 0 & & & $5(16.7 \%)$ \\
\hline Class 1 & $143(67.5 \%)$ & $138(75.8 \%)$ & $6(20.0 \%)$ \\
\hline Class 2 & $38(17.9 \%)$ & $32(17.6 \%)$ & $19(63.3 \%)$ \\
\hline
\end{tabular}


Table 3. Correlations between the investigated parameters

\begin{tabular}{|c|c|c|c|c|c|c|c|c|}
\hline & & Age & Saturation & LOHS (day) & CURB-65 & BCRSS & CALL & PSI \\
\hline \multirow[t]{2}{*}{ Age } & $\mathrm{p}$ & - & 0 & 0 & 0 & 0 & 0 & 0 \\
\hline & $\mathrm{r}$ & & -0.565 & 0.658 & 0.646 & 0.445 & 0.715 & 0.911 \\
\hline \multirow[t]{2}{*}{ Saturation } & $\mathrm{p}$ & 0 & & 0 & 0 & 0 & 0 & 0 \\
\hline & $\mathrm{r}$ & -0.565 & & -0.65 & -0.61 & -0.619 & -0.606 & -0.63 \\
\hline \multirow{2}{*}{ LOHS (days) } & $\mathrm{p}$ & 0 & 0 & & 0 & 0 & 0 & 0 \\
\hline & $\mathrm{r}$ & 0.658 & -0.65 & & 0.621 & 0.666 & 0.75 & 0.696 \\
\hline \multirow[t]{2}{*}{ CURB-65 } & $\mathrm{p}$ & 0 & 0 & 0 & & 0 & 0 & 0 \\
\hline & $r$ & 0.646 & -0.61 & 0.621 & & 0.574 & 0.68 & 0.722 \\
\hline \multirow[t]{2}{*}{ BCRSS } & $\mathrm{p}$ & 0 & 0 & 0 & 0 & & 0 & 0 \\
\hline & $\mathrm{r}$ & 0.445 & -0.619 & 0.666 & 0.574 & & 0.619 & 0.581 \\
\hline \multirow[t]{2}{*}{ CALL } & $\mathrm{p}$ & 0 & 0 & 0 & 0 & & & 0 \\
\hline & $\mathrm{r}$ & 0.715 & -0.606 & 0.75 & 0.68 & 0.619 & & 0.734 \\
\hline \multirow[t]{2}{*}{ PSI } & $\mathrm{p}$ & 0 & 0 & 0 & 0 & & 0 & \\
\hline & $\mathrm{r}$ & 0.911 & -0.63 & 0.696 & 0.722 & 0.581 & 0.734 & \\
\hline
\end{tabular}

LOHS; length of hospital stay

Table 4.Receiver operating characteristic analysis of CURB-65, BCRSS, CALL, and PSI forthe prediction of 30-day mortality

\begin{tabular}{|l|c|c|c|c|c|c|}
\hline & Cut-off value & Sensitivity & Specificity & $\mathbf{9 5 \%}$ CI & AUC & p \\
\hline Age & $>67$ & 76.7 & 91.8 & $0.948-0.798$ & 0.874 & 0.000 \\
\hline Respiratory rate & 19 & 83.3 & 72.68 & $0.916-0.731$ & 0.823 & 0.000 \\
\hline Saturation & $<94$ & 73.3 & 94 & $0.939-0.761$ & 0.850 & 0.000 \\
\hline Length of hospital stay (days) & $>0$ & 90 & 63.9 & $0.874-0.706$ & 0.790 & 0.000 \\
\hline CURB-65 & $>1$ & 83.3 & 86.9 & $0.957-0.762$ & 0.859 & 0.000 \\
\hline BCRSS & $>0.50$ & 80 & 84.7 & $0.944-0.764$ & 0.854 & 0.000 \\
\hline CALL & 8.5 & 80 & 85.2 & $0.947-0.772$ & 0.860 & 0.000 \\
\hline PSI & $>101$ & 80 & 85.2 & $0.972-0.828$ & 0.900 & 0.000 \\
\hline
\end{tabular}

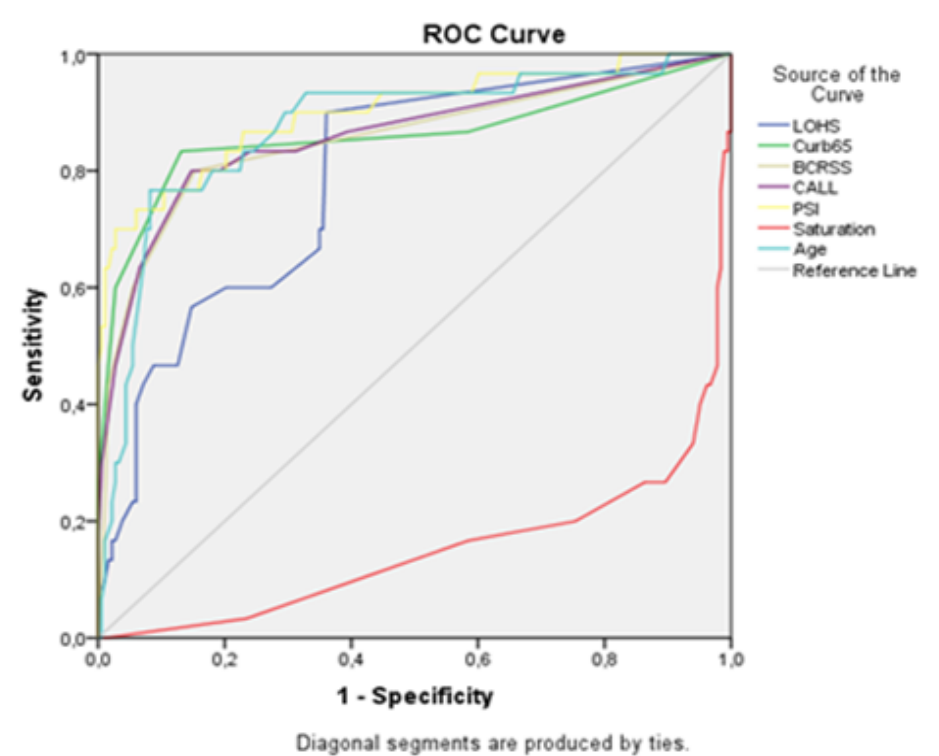

Fig.1. ROC analysis of PSI, CURB-65, BCRSS, CALL, length of hospital stays, age, and saturation in the prediction of mortality

\section{Discussion}

In our study, a statistically significant relationship was found between the PSI, CURB-65, CALL and BCRSS scores and mortality in patients withCOVID-19, and it was determined that PSI was superior to the other scoring systems in predicting mortality in this patient group. Although the literature contains studies comparing different classifications, to the best of our knowledge, our study is the first to compare PSI, Curb-65, CALL and BCRSS in the prediction of prognosis in patients with COVID-19.

In studies comparing scoring systems in COVID-19 patients, the effects of comorbidity and symptoms on mortality were also evaluated. Kamran et al. suggested that comorbidity was not area under the curve: $(p=0.565)$ for mortality (7) while another study evaluating 52 intensive care patients with COVID-19 reported a relationship between advanced age and comorbidities and mortality (8). Zhang et al., examined 80 patients and found a significant relationship between cardiac diseases and hypertension and mortality (9) whereas Zhu et al. retrospectively observed that among 181 patients with COVID-19, comorbidities were not predictive of mortality (10). In the same study, it was 
revealed that the symptoms of cough, shortness of breath and diarrhea were statistically significantly associated with mortality (10). In a study conducted in Wuhan, Zhou et al. determined the most common symptoms as fever and cough in 191 patients and noted that mortality was higher in patients with diabetes mellitus and coronary artery disease (11). In our study, while our patients most frequently presented with a cough, mortality was significantly higher in those with the comorbidities of CHF, HT, COPD, CKF and CAD. Confusion, cough ( $<<0.001)$, myalgia and sore throat ( $p<0.024$ ) were found to be statistically significant in determining mortality.

In the prediction of prognosis and mortality using scoring systems, not only symptoms and comorbidities but also examination findings and laboratory test results can contribute to this evaluation. In a study examining 419 patients with community-acquired pneumonia, it was emphasized that PSI better defined a low risk of death while CURB-65 was able to show a high risk of death but did not have any prediction about comorbidities (12). In patients diagnosed with COVID-19, different scoring systems have been used, and their superiority in predicting mortality differed. For example, in a prospective study evaluating 249 patients with COVID-19, Garcia-Clemente et al. found that PSI and CURB-65 were determinants of mortality (13). In another study comparing laboratory parameters, as well as scoring systems, there was a statistically significant relationship between the CURB-65, PSI and COVID-19 severity scores and length of hospital stay, and the authors noted that CURB-65 and PSI outperformed the COVID-19 severity score in predicting mortality, but they were not superior to each other (10). In a retrospective classification study conducted by Fan et al. in COVID-19 cases, it was stated that CURB-65 and PSI did not have significant advantages over each other in predicting mortality (14). In our study, a statistically significant relationship was found between PSI and CURB-65 and mortality, and PSI was superior to CURB-65 in this evaluation. Both PSI and CURB-65 were positively correlated with the length of hospital stay.

In addition to the previously used scoring systems, different classification systems have been introduced to evaluate prognosis and predict mortality in COVID-19. BCRSS was developed by the Italian Society of Infectious and Tropical Diseases to determine patients that should be given tocilizumab treatment. This score was applied to the patients with a high risk of COVID-19 and those with a positive COVID-19 PCR result. Accordingly, it was determined that BCRSS should be $\geq 3(5,15)$. In a study showing that tocilizumab treatment could prevent acute lung injury in patients with COVID-19, BCRSS was used as a guide to show the necessity and efficacy of treatment. In that study, it was determined that the coexistence of BCRSSguided treatment and a low comorbidity rate resulted in reduced mortality (16). And it was also reported that BCRSS ensured that this treatment was effective in the early period (17). However, there are very few studies comparing BCRSS with other scoring systems. In a retrospective study in which 313 patients with COVID-19 were examined, it was found that CURB-65 had higher ability to determine in-hospital mortality compared to BCRSS while the latter was a better predictor of intensive care requirement (18). In contrast, in our study, both BCRSS and CURB-65 showed a positive correlation with the length of hospital stay, and neither was superior to the other in predicting mortality.

The CALL scoring system involves the evaluation of comorbidities, age, lymphocyte, and lactate dehydrogenase, which have been emphasized to play a role in COVID-19 since the emergence of the disease, and studies have been conducted to investigate the effect of these parameters on mortality. In a study examining 252 patients with COVID19, it was shown that CALL was a reliable model for predicting mortality and determining the progression of the disease (7). In another study, Grifoni et al. similarly determined that CALL was a reliable model for predicting mortality in patients with COVID-19 but noted that it did not show disease progression at a sufficient level (19). In the current study, CALL was positively correlated with the length of hospital stay and presented as a reliable model for predicting mortality.

In our study, the four scoring systems were positively correlated with each other and with the length of hospital stay. However, the length of hospital stay was relatively weaker in predicting mortality compared to the scoring systems evaluated in our study. This can be explained by the rapid progression of the disease with the presence of comorbidities and increasing age. However, despite its weak predictive ability, the length of hospital stay was still statistically significantly correlated with mortality. Therefore, PSI, CURB-65, BCRSS and CALL also showing a positive correlation with the length of hospital further confirm that these scoring systems are determinants of both mortality and progression in patients with COVID-19.

This study was conducted in a single center with patients that had a positive PCR test. Although this test is accepted worldwide, some of our patients with two or three negative PCR test results, who were followed up for symptoms, were determined to have CT findings compatible with COVID-19. This patient group was not included in the study because their PCR test was negative.

Scoring systems are especially important in predicting the progression of COVID-19. Scoring systems, which include comorbidity and vital signs as well as laboratory and imaging findings, will be more effective than many scoring systems in determining the prognosis and mortality in patients with COVID-19. The comparative data of scoring systems will contribute to the literature in terms of taking the 
Akça et al. / J Exp Clin Med

necessary precautions, intervening in a timely manner, and making follow-up decisions in the hospital. There is a need for meta-analyses using different scoring systems and comparing the data obtained from different countries. This will assist in determining a common algorithm and achieve reduced mortality and length of hospital stay.

\section{References}

1. Republic of Turkey Ministry of Health. Covid-19 Information page. 2020 (Updated 2020 mar 11; cited 2021 Feb 16). Available from: https:/covid19bilgi.saglik.gov.tr/depo/rehberler/COVID-19_Rehberi.pdf.

2. Lim WS, van der Eerden MM, Laing R, Boersma WG, Karalus N, Town GI et al. Defining community acquired pneumonia severity on presentation to hospital: an international derivation and validation study. Thorax 2003; 58:377-82.

3. Barlow G, Nathwani D, Davey P. The CURB65 pneumonia severity score outperforms generic sepsis and early warning scores in predicting mortality in community-acquired pneumonia. Thorax 2007;62(3):253-9. Doi: 10.1136/thx.2006.067371.

4. Ji D, Zhang $\mathrm{D}$, Xu J, Chen $\mathrm{Z}$, Yang $\mathrm{T}$, Zhao $\mathrm{P}$ et al. Prediction for Progression Risk in Patients with COVID19 Pneumonia: The CALL Score. Clin Infect Dis 2020;71(6):1393-9. Doi: 10.1093/cid/ciaa414.

5. Duca A, Piva S, Foca E, Latronico N, Rizzi M. Calculated Decisions: Brescia-COVID Respiratory Severity Scale $($ BCRSS)/Algorithm. $)=6$. Emerg Med Pract 2020; 16:22(5 Suppl):CD1-CD2.

6. Clinical management of COVID-19. 2020 (Updated 2020 mar 11; cited 2021 Feb 16). Available from: https://www.who.int/publications/i/item/clinical-managementof-covid-19.

7. Kamran S M, Mirza Z, Moeed H, Naseem A, Hussain $\mathrm{M}$, Fazal Sr I et al. CALL Score and RAS Score as Predictive Models for Coronavirus Disease 2019. Cureus 2020;12(11): e11368. Doi:10.7759/cureus.11368.

8. Yang X, Yu Y, Xu J, Shu H, Xia J, Liu H et al.Clinical course and outcomes of critically ill patients with SARS-CoV-2 pneumonia in Wuhan, China: a single-centered, retrospective, observational study. Lancet Respir Med 2020; 8:475-81.

9. Zhang C, Qin L, Li K, Wang Q, Zhao Y, Xu B et al. A novel scoring system for prediction of disease severity in COVID19. Front Cell Infect Microbiol 2020; 10:318. Doi:10.3389/fcimb.2020.00318.
10. Zhu JS, Ge P, Jiang C, Zhang Y, Li X, Zhao Z et al. Deep learning artificial intelligence analysis of clinical variables predicts mortality in COVID 19 patients. Infectious Disease. $\begin{array}{llll}\text { JACEP } & \text { Open } & \text { 2020; }\end{array}$ 73. https://doi.org/10.1002/emp2.12205.

11. Zhou F, Yu T, Du R, Fan G, Liu Y, Liu Z et al. Clinical course and risk factors for mortality of adult in patients with COVID-19 in Wuhan, China: a retrospective cohort study. Lancet 2020; 395:1054-62. Doi:10.1016/S01406736(20)30566-3.

12. Menéndez R, Martinez R, Reyes $S$, Mensa J, Filella $X$, Marcos MA et al. Biomarkers improve mortality prediction by prognostic scales in community-acquired pneumonia. Thorax 2009; 64:587-91. Doi:10.1136/thx.2008.105312.

13. Garcia Clemente MM, Huertas JH, Fernandez AF, Escosura Munoz C, Enriquez Rodriguez AI, Martinez LP et al. Assessment of risk scores in Covid 19. Int J Clin Pract. 2020;00: e13705. https://doi.org/10.1111/ijcp.13705.

14. Fan $\mathrm{G}, \mathrm{Tu} \mathrm{C}$, Zhou F, Liu $\mathrm{Z}$, Wang $\mathrm{Y}$, Song $\mathrm{B}$ et al. Comparison of severity scores for COVID-19 patients with pneumonia: a retrospective study. Eur Respir J 2020; 56:2002113. Doi: 10.1183/13993003.02113-2020.

15. Lombardy Section Italian Society Infectious and Tropical Diseases. Vademecum for the treatment of people with COVID 19. Edition 2.0, 13 March 2020. Infez Med 2020;28(2):143-52.

16. Toniati P, Piva S, Cattalini M, Garrafa E, Regola F, Castelli F et al. Tocilizumab for the treatment of severe COVID-19 pneumonia with hyperinflammatory syndrome and acute respiratory failure: A single center study of 100 patients in Brescia, Italy. Autoimmunity Reviews 2020;19(7):102568. https://doi.org/10.1016/j.autrev.2020.102568.

17. Moreno-Pérez O, Andres M, Leon-Ramirez J-M, SánchezPayá J, Rodríguez JC, Sánchez R et al. Experience with tocilizumab in severe COVID-19 pneumonia after 80 days of follow-up: A retrospective cohort study, J Autoimmun 2020;114:102523. https://doi.org/10.1016/j.jaut.2020.102523.

18. Rodriguez-Nava G, Yanez-Bello MA, Trelles-Garcia DP, Chung CW, Friedman HJ, Hines DW. Performance of the quick COVID-19 severity index and the Brescia-COVID respiratory severity scale in hospitalized patients with COVID-19 in a community hospital setting. IJID 2021;102: 571-6. https://doi.org/10.1016/j.ijid.2020.11.003.

19. Grifoni E, Valoriani A, Cei F, Vannucchi V, Moroni F, Pelagatti L et al. The CALL score for predicting outcomes in patients with COVID-19. Clin Infect Dis 2020. Doi:10.1093/cid/ciaa686. 